$$
\text { CONF-970706--5 }
$$

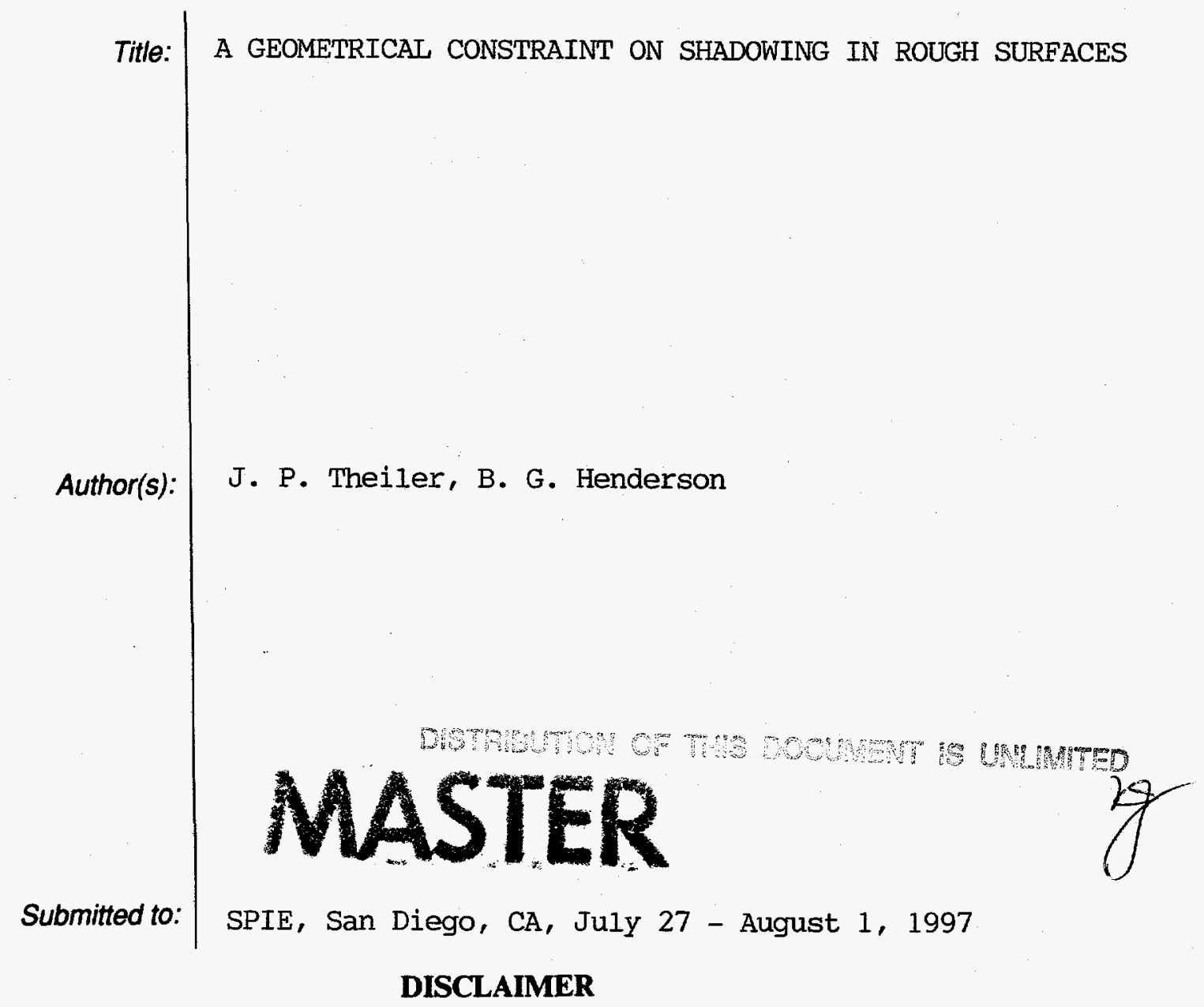

This report was prepared as an account of work sponsored by an agency of the United States Government. Neither the United States Government nor any agency thereof, nor any of their employees, makes any warranty, express or implied, or assumes any legal liability or responsibility for the accuracy, completeness, or usefulness of any information, apparatus, product, or process disclosed, or represents that its use would not infringe privately owned rights. Reference herein to any specific commercial product, process, or service by trade name, trademark, manufacturer, or otherwise does not necessarily constitute or imply its endorsement, recommendation, or favoring by the United States Government or any agency thereof. The views and opinions of authors expressed herein do not necessarily state or reflect those of the United States Government or any agency thereof.

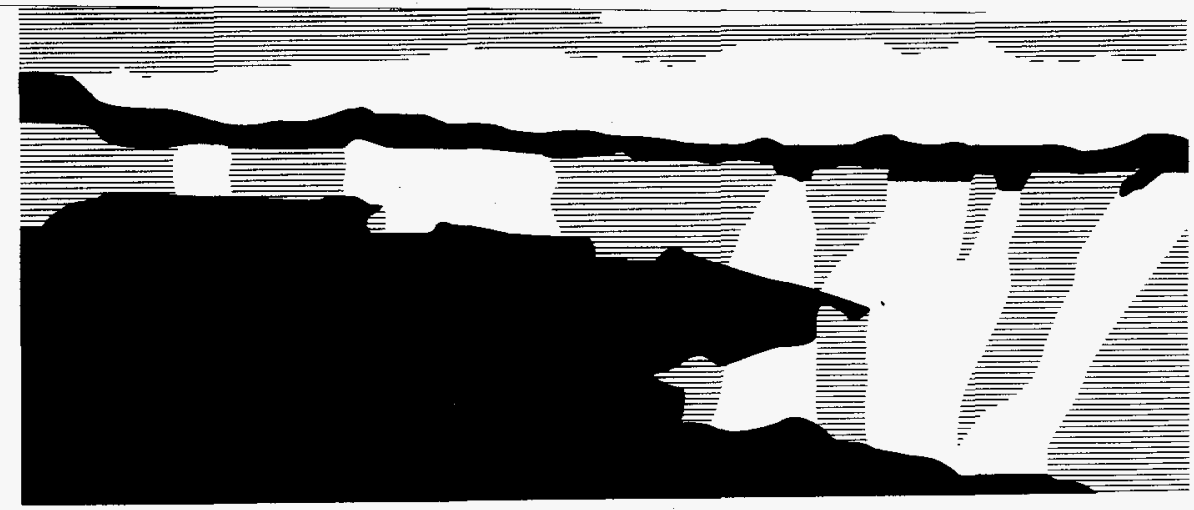

Los Alamos National Laboratory, an affirmative action/equal opportunity emplóyer, is operated by the University of California for the U.S. Department of Energy under contract W-7405-ENG-36. By acceptance of this article, the publisher recognizes that the U.S. Government retains a nonexclusive, royalty-free license to under contract W-740 5-ENG-36. By acceptance of this article, the pllow others to do so, for U.S. Government purposes. The Los Alamos National Laboratory requests that the publisher identify this article as work performed under the auspices of the U.S. Department of Energy. 


\section{DISCLAMIER}

Portions of this doeument may be illegible in electronic image products. Images are produced from the best available original docoment. 


\title{
A Geometrical Constraint on Shadowing in Rough Surfaces
}

\author{
James Theiler and Bradley G. Henderson \\ Astrophysics and Radiation Measurements Group \\ Los Alamos National Laboratory \\ Los Alamos, New Mexico 87545 USA
}

\begin{abstract}
By considering a one-dimensional cross-section through the rough surface, we derive a purely geometrical constraint on the statistical distribution of shadowed facet slopes that should be satisfied by any model of surface emissivity that includes the effect of self-shadowing. Our purpose is not to develop a single shadowing model, but to provide a condition that any valid shadowing model should satisfy. Although the emphasis of the presentation is theoretical, some practical ramifications will also be discussed.
\end{abstract}

Keywords: emissivity, shadowing, rough surface, view angle, model

\section{INTRODUCTION}

In modeling the thermal emissivity of a rough surface, such as wind-driven waves on a lake or ocean, it is customary to treat the surface as a collection of individual "facets", each of which is flat but tilted with respect to the horizontal. (This is valid because the characteristic size of ocean capillary waves is on the order of a centimeter, ${ }^{1}$ which is much larger than the wavelengths of radiation we are interested in.) To first order, the facets can be treated independently, and the total emissivity of the rough surface is obtained by adding together the individual emissivities of the facets. However, there are also higher order effects that should be considered, particularly when the surface is viewed from an angle that is far from vertical. These higher order effects include multiple reflection, polarization, and shadowing.

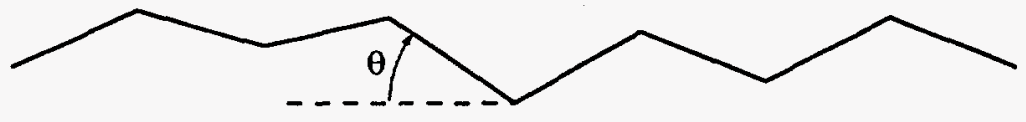

Figure 1. A rough surface, seen in cross section, is composed of flat "facets" at angles $\theta$ to the horizontal.

By considering a one-dimensional cross-section through the rough surface, it is possible to derive a purely geometrical constraint that should be satisfied by any model for shadowing. This constraint, given in Eq. (17), applies to all one-dimensional cross sections. In this one-dimensional cross-section, a facet becomes a simple line segment which is tilted at some angle $\theta$ to the horizontal. A flat surface, such as still water, will have $\theta=0$. Note that $\theta$ can be positive or negative, and can in principle vary from $-\pi$ to $\pi$, though $|\theta|>\pi / 2$ requires an overhang. Let $P(\theta)$ be the distribution of facet angles in the surface, so that $P(\theta) \mathrm{d} \theta$ is the probability that a random vertical ray of light will intersect a facet whose angle is between $\theta$ and $\theta+\mathrm{d} \theta$. If all facets have equal area (as viewed from the vertical), then $P(\theta) \mathrm{d} \theta$ will also be the probability that a randomly chosen facet with have an angle between $\theta$ and $\theta+\mathrm{d} \theta$.

While $P(\theta)$ obviously is not a full description of a rough faceted surface, it provides enough information for a first order model of emissivity as a function of look angle. A model that also includes shadowing will have to provide further information. The purpose of this report is to derive a constraint on the additional information that a shadowing model can provide.

Work by both authors supported by the U.S. Department of Energy. Email: \{jt,bghenderson\} $\bullet 1$ anl.gov. 


\section{FIRST ORDER MODEL}

Since $P(\theta)$ is a probability distribution, we can write

$$
\int_{-\pi}^{\pi} P(\theta) \mathrm{d} \theta=1
$$

Further, since there can be no overall slope to the surface, we also require

$$
\langle\tan \theta\rangle=\int_{-\pi}^{\pi} \tan \theta P(\theta) \mathrm{d} \theta=0 .
$$

Let us write $\varepsilon_{o}(\omega)$ as the emissivity of a facet as viewed from the angle $\omega$ with respect to that facet's normal. Note that $\varepsilon_{o}(\omega)$ is an intrinsic property of the surface material, and not of the surface roughness. In practice, $\varepsilon_{o}(\omega)$ will be polarization dependent and will be given by the Fresnel relations (see for instance Ref. 2, pp. 280-282). Fig. 2 shows a representative Fresnel emissivity $\varepsilon_{o}(\omega)$ from a flat facet as a function of the emission angle $\omega$; this emissivity is for water observed in the infrared at four microns, using index of refraction data from Irvine and Pollack. ${ }^{3}$ And let us write $\varepsilon(0)$ - without the subscript naught - as the effective emissivity of the rough surface (again, neglecting multiple reflections and shadowing) as seen from the vertical:

$$
\varepsilon(0)=\int_{-\pi}^{\pi} \varepsilon_{o}(\theta) P(\theta) \mathrm{d} \theta
$$

For an ideal Lambertian facets with $\varepsilon_{o}(\omega)=1$, the rough surface will also be ideal Lambertian with an effective emissivity $\epsilon(0)=1$.

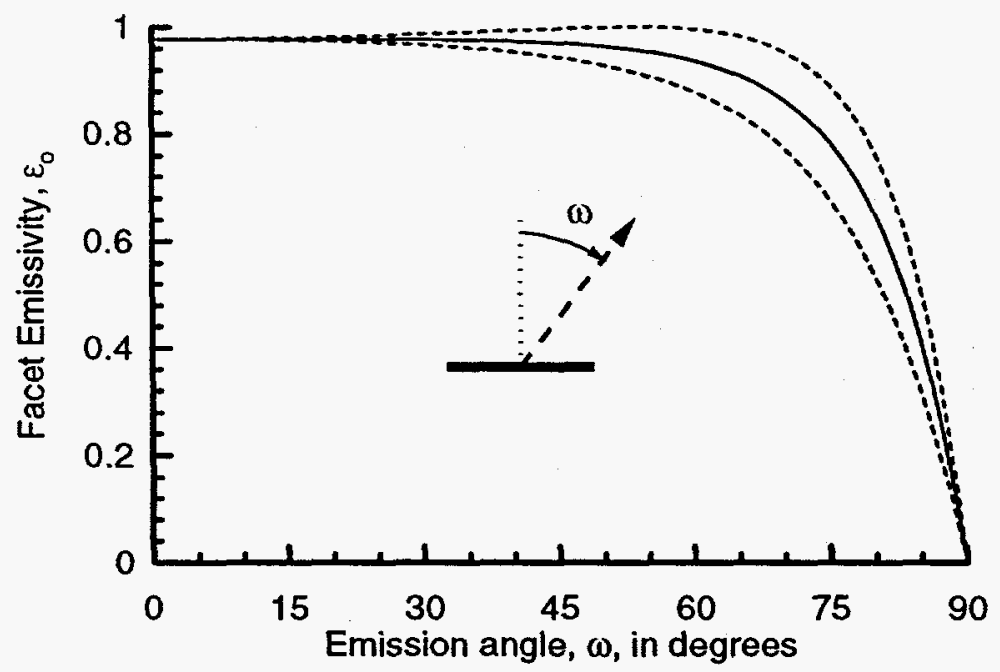

Figure 2. Emissivity $\varepsilon_{o}(\omega)$ of a single flat facet as a function of the emission angle $\omega$. The dotted lines are for polarized light: the upper curve is parallel, and the lower curve is perpendicular. The solid line is for unpolarized light. These Fresnel emissivity curves are based on the index of refraction for water observed in the infrared at $4 \mu \mathrm{m}$.

\subsection{Nonvertical lookangle}

Now, let us observe this surface from an angle $\phi$ from the vertical. Then the facet which had an angle $\theta$ as observed from the vertical will have angle $\theta-\phi$ as observed from lookangle $\phi$. So the projected size of the facet will be a factor of $\frac{\cos (\theta-\phi)}{\cos \theta}$ larger (or smaller if the factor is less than one) when seen at the look angle $\phi$ compared to its size as seen from the vertical. Since we are looking at an angle $\phi$, we will see a factor of $1 / \cos \phi$ more of the surface (that many more facets) for a given angular field of view. Combining these two effects gives a new probability distribution

$$
P(\phi ; \theta)=\frac{\cos (\theta-\phi)}{\cos \theta \cos \phi} P(\theta)
$$




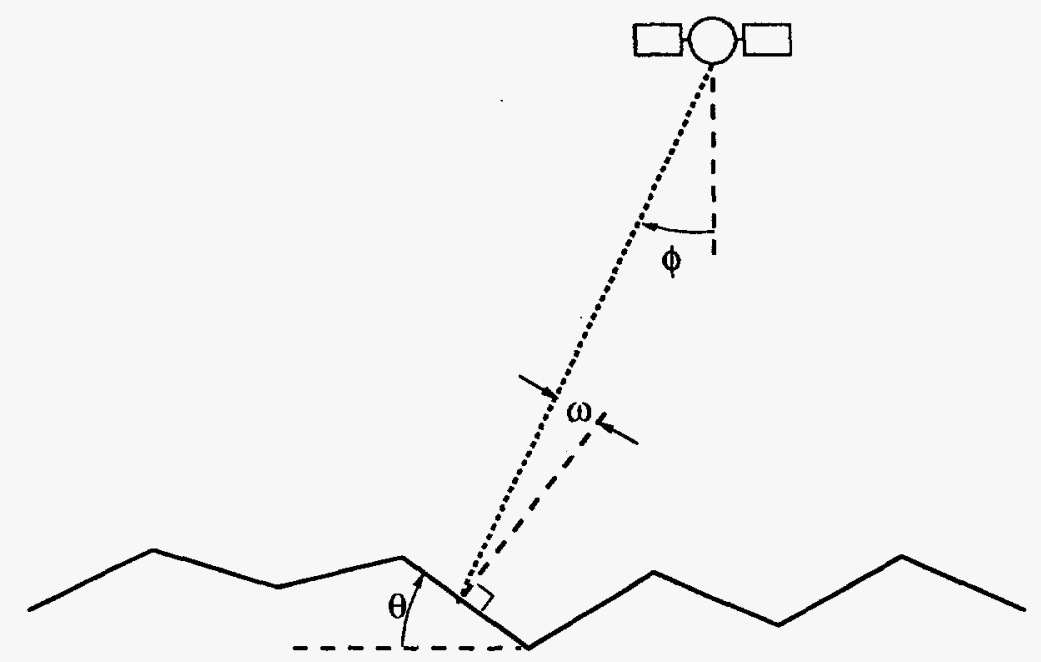

Figure 3. Rough surface is observed from a look angle $\phi$. The emission angle $\omega=\theta-\phi$ is the angle from the look direction to the facet normal.

that a random ray of light emanating at an angle $\phi$ will have departed from a facet of the surface whose angle with respect to the horizontal is $\theta$. Expanding the $\cos (\theta-\phi)$ term, we can rewrite this equation as

$$
P(\phi ; \theta)=(1+\tan \theta \tan \phi) P(\theta) .
$$

Note that the arguments to this point have been completely geometrical. Using only $P(\theta)$ as our "input" information, we have derived a more general distribution $P(\phi ; \theta)$.

By the way, it is a straightforward mathematical identity that

$$
\begin{aligned}
\int_{-\pi}^{\pi} P(\phi ; \theta) \mathrm{d} \theta & =\int_{-\pi}^{\pi}(1+\tan \theta \tan \phi) P(\theta) \mathrm{d} \theta \\
& =\int_{-\pi}^{\pi} P(\theta) \mathrm{d} \theta+\tan \phi \int_{-\pi}^{\pi} \tan \theta P(\theta) \mathrm{d} \theta \\
& =1,
\end{aligned}
$$

so that $P(\phi ; \theta)$ behaves like a probability distribution ${ }^{4}$ in $\theta$. Again, using $\varepsilon_{o}(\omega)$ as the emissivity of a flat facet as observed from the angle $\omega$, we can obtain the effective emissivity of this rough surface by

$$
\begin{aligned}
\varepsilon(\phi) & =\int_{-\pi}^{\pi} \varepsilon_{o}(\phi-\theta) P(\phi ; \theta) \mathrm{d} \theta \\
& =\int_{-\pi}^{\pi} \varepsilon_{o}(\phi-\theta)(1+\tan \phi \tan \theta) P(\theta) \mathrm{d} \theta \\
& =\int_{-\pi}^{\pi} \varepsilon_{o}(\phi-\theta) P(\theta) \mathrm{d} \theta+\tan \phi \int_{-\pi}^{\pi} \varepsilon_{o}(\phi-\theta) \tan \theta P(\theta) \mathrm{d} \theta
\end{aligned}
$$

the first term of which is a convolution of the flat-surface emissivity function with the distribution $P(\theta)$; the second term will be small if the look angle $\phi$ is small, or if $\varepsilon_{o}(\omega)$ is nearly constant. Again, we see that to first order (i.e., neglecting multiple reflections and shadowing), we can derive the effective emissivity $\varepsilon(\phi)$ of the full rough surface using as input only the flat-surface emissivity $\varepsilon_{o}(\omega)$ (this is the "physics") and the distribution $P(\theta)$ (this is the "geometry").

Again, note that if the individual facets are Lambertian, so that $\varepsilon_{o}(\omega)=1$, then the above equation implies that $\varepsilon(\phi)=1$; that is, that the rough surface will also be Lambertian. Note that this is really a statement about the geometry of the surface, and not about the physics of the actual emissivity. 


\section{SHADOWING}

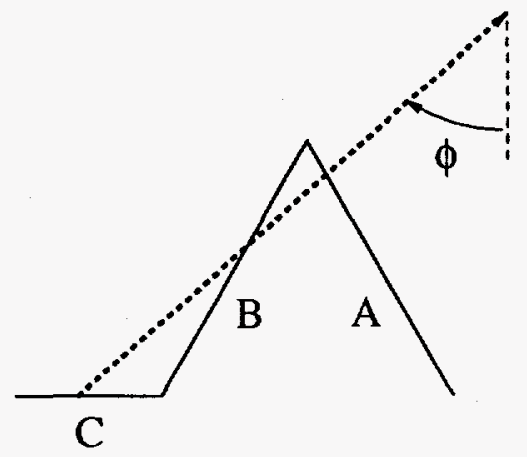

Figure 4. Three kind of facets are shown: type $\mathrm{A}$ is visible from the lookangle $\phi$, type $\mathrm{B}$ is angled backward with respect to the lookangle, and type $\mathrm{C}$ is shadowed by facets of type $\mathrm{B}$.

If a surface is rough enough, and if the lookangle $\phi$ is large enough, then some of the surface will be in shadow. The condition for some shadowing to occur is that there be some facets which are angled backward with respect to the look angle. That is, that $P(\theta)>0$ for some $\theta$ satisfying $|\phi-\theta|>\pi / 2$. In this case, the surface can be partitioned into three kinds of facets:
A : facet is fully visible from look angle $\phi$
B : facet makes a backward angle with respect to look angle (Note, this occurs if $|\theta-\phi|>\pi / 2$ ).
$\mathrm{C}$ : facet is blocked ("shadowed") from view by a facet of type B.

To fully specify a shadowing model, we can write probability distributions $P_{A}, P_{B}$, and $P_{C}$, corresponding to the three kinds of facets, and satisfying

$$
P(\phi ; \theta)=P_{A}(\phi ; \theta)+P_{B}(\phi ; \theta)+P_{C}(\phi ; \theta) .
$$

Combining with Eq. (5), we obtain

$$
(1+\tan \theta \tan \phi) P(\theta)=P_{A}(\phi ; \theta)+P_{B}(\phi ; \theta)+P_{C}(\phi ; \theta) .
$$

But there is a problem here. The left-hand-side of this equation (whose right hand side is a sum of probabilities) becomes negative when $|\phi-\theta|>\pi / 2$. It might be tempting to simply redefine the expression for $P(\phi ; \theta)$ given in Eq. (5), for instance by replacing it with its absolute value, but the integral in Eq. (6) would then be larger than unity. If we treat $P(\phi ; \theta)$ not as a formal probability, but instead as a kind of "rate" at which rays cross the surface, then we no longer require that the identity in Eq. (6) be satisfied. For a flat surface, this rate integrates to 1, but when shadowing is present, it becomes possible for a ray to intersect the surface more than once.

A more useful interpretation is to "sign" the intersections as positive or negative, according to whether the ray intersects the facet from above or from below. In this case the sum of the intersections of a ray with a surface, no matter how rough, is always one. Now, we have an interpretation for the negative $P(\phi ; \theta)$ that appears in Eq. (5) when $|\phi-\theta|>\pi / 2$; these correspond to "negative" crossings. And furthermore, we recover the identity in Eq. (6).

To be a little more precise, we intepret $P_{X}(\phi ; \theta)$ as the idealized $\varepsilon_{o}=1$ Lambertian emissivity of the surface due to the type $X$ facets. And in this mathematical idealization, we say that back-angled facets contribute a negative emissivity!

It bears emphasizing that, unlike what we were able to do in Eq. (5), we cannot "derive" the individual $P_{A}(\phi ; \theta)$ or $P_{C}(\phi ; \theta)$ terms just from $P(\theta)$ and geometrical considerations; however we can derive $P_{B}(\phi ; \theta)$. Since type $\mathrm{B}$ facets occur only for the back-angles, we have

$$
P_{B}(\phi ; \theta)= \begin{cases}(1+\tan \theta \tan \phi) P(\theta) & \text { for }|\phi-\theta|>\pi / 2 \\ 0 & \text { otherwise }\end{cases}
$$


Recall that $P(\phi ; \theta)$ is negative if and only if $|\phi-\theta|>\pi / 2$. So we have that $P_{B}(\phi ; \theta)$ is always zero or negative, while $P_{A}(\phi ; \theta)$ and $P_{C}(\phi ; \theta)$ are always non-negative.

Let us introduce the "shadowing fraction" $S(\phi ; \theta)$ which gives the fraction of $\theta$-facets that are in shadow:

$$
S(\phi ; \theta)=\frac{P_{C}(\phi ; \theta)}{P_{A}(\phi ; \theta)+P_{C}(\phi ; \theta)}
$$

for $|\phi-\theta| \leq \pi / 2$, and $S(\phi ; \theta)=1$ for back-angled facets: $|\phi-\theta|>\pi / 2$.

Physically, we know that only the type A facets contribute to the effectivity emissivity of the surface, so we can write

$$
\varepsilon(\phi)=\int_{-\pi}^{\pi} \varepsilon_{o}(\phi-\theta) P_{A}(\phi ; \theta) \mathrm{d} \theta
$$

Under the mathematical ideal of Lambertian emission with $\varepsilon_{o}=1$, we have

$$
1=\varepsilon_{A}(\phi)=\int_{-\pi}^{\pi} P_{A}(\phi ; \theta) \mathrm{d} \theta .
$$

This provides an important constraint on the function $P_{A}(\phi ; \theta)$, and therefore on the shadowing model, as specified by $S(\phi ; \theta)$.

Since $P_{A}(\phi ; \theta)+P_{C}(\phi ; \theta)=P(\phi ; \theta)$ for $|\phi-\theta| \leq \pi / 2$, and $S(\phi ; \theta)=1$ for $|\phi-\theta|>\pi / 2$, we can write

$$
P_{A}(\phi ; \theta)=(1-S(\phi ; \theta)) P(\phi ; \theta)
$$

for all $\phi$ and $\theta$; and so the constraint in Eq. (15) can be combined with the formula for $P(\phi ; \theta)$ in Eq. (5), and the identity in Eq. (6) to provide the main result: a constraint on $S(\phi ; \theta)$.

$$
\int_{-\pi}^{\pi} S(\phi ; \theta)(1+\tan \theta \tan \phi) P(\theta) \mathrm{d} \theta=0 .
$$

Note that although we are treating the facets as if they were Lambertian facets, our results do not depend on this assumption; rather, the Lambertian ideal is a convenient tool for making "physical" the properties that are ultimately geometrical.

Finally, we note that the surface emissivity at look-angle $\phi$ is given by

$$
\varepsilon(\phi)=\int_{-\pi}^{\pi} \varepsilon_{o}(\phi-\theta)(1-S(\phi ; \theta))((1+\tan \phi \tan \theta) P(\theta)) \mathrm{d} \theta .
$$

\section{EXAMPLES OF LEGAL AND ILLEGAL SHADOWING MODELS}

The simplest shadowing model is to have no shadowing at all. That is $S(\phi ; \theta)=0$. This is in fact the first-order model described previously, and is completely "valid" insofar as the constraint in Eq. (17) is satisfied, but of course it is inaccurate if there are any back-angled facets.

An almost-as-simple model can arise inadvertently in the implementation of the first-order model, by simply resetting $P(\phi ; \theta)$ to zero whenever it is negative. That is, let

$$
P^{\prime}(\phi ; \theta)=\max (0, P(\phi ; \theta))
$$

and use this $P^{\prime}(\phi ; \theta)$ in place of $P(\phi ; \theta)$ in Eq. (7) for the rough surface emissivity. Since $P(\phi ; \theta)$ is "like" a probability, this is a seemingly reasonable approach. It essentially says that only the back-angled facets are shadowed, however, and neglects the shadowing of facets of type C. In other words,

$$
S(\phi ; \theta)= \begin{cases}1 & \text { for }|\phi-\theta|>\pi / 2 \\ 0 & \text { otherwise }\end{cases}
$$


It is not hard to show that this violates the constraint in Eq. (17), and is therefore an invalid shadowing model. This model will invariably overestimate the emissivity of the surface because it ignores the type C facets. Some authors have taken this as the "unshadowed" model, and have been concerned that it diverges as $\phi \rightarrow \pi / 2$; that is, as one looks to the horizon. The role of shadowing on the properties of the ocean-sky horizon (and indeed its very existence $^{5}$ ) continue to interest researchers. ${ }^{1,6}$

The notion of treating $P(\phi ; \theta)$ as a probability suggests another very simple shadowing model. Having set $P^{\prime}(\phi ; \theta)=0$ where $P(\phi ; \theta)$ was previously negative, we no longer have that the integral of $P^{\prime}(\phi ; \theta)$ is unity. The easiest way to fix this up is just to rescale the probability. That is,

$$
P^{\prime \prime}(\phi ; \theta)=\frac{P^{\prime}(\phi ; \theta)}{\int_{-\pi}^{\pi} P^{\prime}(\phi ; \theta) \mathrm{d} \theta}
$$

Again, without too much difficulty, it can be shown that this is equivalent to writing

$$
S(\phi ; \theta)= \begin{cases}1 & \text { for }|\phi-\theta|>\pi / 2 \\ 1-\frac{1}{\int_{-\pi}^{\pi} P^{\prime}(\phi ; \theta) \mathrm{d} \theta} & \text { otherwise }\end{cases}
$$

and that this "model" satisfies the geometric criterion derived in the previous section. A noteworthy feature of this model is that $S(\phi ; \theta)$ depends only on $\phi$, and is independent of $\theta$. That is, all facets are shadowed equally likely. It is also noteworthy that this model is specified completely from the $P(\theta)$ function.

Interestingly, this "very simple" shadowing model has been derived earlier in two quite different ways. Wagner ${ }^{7}$ takes a very particular model of surface roughness, and derives an analytical expression for $S(\phi ; \theta)$ which turns out to be independent of $\theta$. Saunders, ${ }^{5}$ by contrast, postulates that $S(\phi ; \theta)$ is independent of $\theta$, and then derives a geometrical constraint that is only slightly less general ${ }^{8}$ than our Eq. (17). What is remarkable about Saunders' result is that the shadowing model $S(\phi ; \theta)$ is complelely specified by the surface roughness $P(\theta)$ and the assumption that $S(\phi ; \theta)$ is independent of $\theta$. In a later paper, Saunders ${ }^{9}$ goes on to use his results to compute infrared radiance of the ocean in the presence of shadowing. The analytical form of $S(\phi ; \theta)$ in the case where $P(\theta)$ is taken to be gaussian in $\tan \theta$ is a rather complicated expression, and it is impressive to see it derived in two different ways in the papers by Wagner ${ }^{7}$ and by Saunders. ${ }^{5}$ If the distribution of facet slopes is more complicated than a gaussian, as for instance given by the experimental results of Cox and Munk, ${ }^{10}$ then an analytical form for $S(\phi ; \theta)$ will be difficult to derive. However, the shadowing model is easy to implement simply by normalizing the probability in Eq. (21).

In fact, normalizing the probability function is the easiest way to enforce the validity of arbitrary shadowing models. For example, let $s(\phi ; \theta)$ represent a "first-cut" shadowing model, one that for instance addresses the issue raised by Saunders ${ }^{5}$ in questioning the validity of the assumption that $S(\phi ; \theta)$ was independent of $\theta$ :

It seems plausible that horizontal slopes and gentle slopes of either sign are shadowed with equal frequency since they will occur with equal frequency in the troughs and crests of waves. On the other hand, it is less plausible and even intuitively doubtful that steep slopes facing the observer are shadowed with equal frequency with the nearly horizontal slopes.

Take $P^{\prime}(\phi ; \theta)$ as in Eq. (19), define

$$
P^{\prime \prime}(\phi ; \theta)=(1-s(\phi ; \theta)) P^{\prime}(\phi ; \theta)
$$

and let

$$
t(\phi ; \theta)=1-\frac{1}{\int_{-\pi}^{\pi} P^{\prime \prime}(\phi ; \theta) \mathrm{d} \theta}
$$

Note that if you were lucky and your $s(\phi ; \theta)$ was a valid shadowing model, then $t(\phi ; \theta)$ would be zero. But if not, then

$$
S(\phi ; \theta)=1-(1-s(\phi ; \theta))(1-t(\phi ; \theta))
$$

will be a valid shadowing model. And it is a model that can be implemented simply by normalizing $P^{\prime \prime}(\phi ; \theta)$

$$
P^{\prime \prime \prime}(\phi ; \theta)=\frac{P^{\prime \prime}(\phi ; \theta)}{\int_{-\pi}^{\pi} P^{\prime \prime}(\phi ; \theta) \mathrm{d} \theta}
$$

and using $P^{\prime \prime \prime}(\phi ; \theta)$ as the probability function in Eq. (7). 


\section{PRELIMINARY NUMERICAL EXPERIMENT}

Since a number of shadowing models posit that the shadowing fraction is independent of facet angle, we did a preliminary numerical experiment to check the validity of this assumption for a particular surface model. This involved a Monte-Carlo simulation (but see Ref. 11 for an alternative, more deterministic approach) using software developed for the more extensive study in Ref. 12. We employed the random capillary facet model of Preisendorfer and Mobley, ${ }^{13,14}$ set the wind speed to $15 \mathrm{~m} / \mathrm{sec}$, and used 5000 random rays (actually, what is randomized are the realizations of the wind-roughened surface) for each value of the lookangle $\phi$. We counted the number of rays that struck surfaces of type ' $\mathrm{A}$ ' and of type ' $\mathrm{C}$ ', and from these estimated the fraction $S(\phi ; \theta)$ defined in Eq. (13). Fig. 5 shows the result of these experiments for four values of $\phi$. The shadowing fraction became negligibly small for $\phi \leq 65^{\circ}$. There is some indication of nonconstant variation of $S(\phi ; \theta)$ with $\theta$; facet angles which are nearly parallel to the incoming rays appear to be somewhat more likely to be shadowed, though the effect is not dramatic.

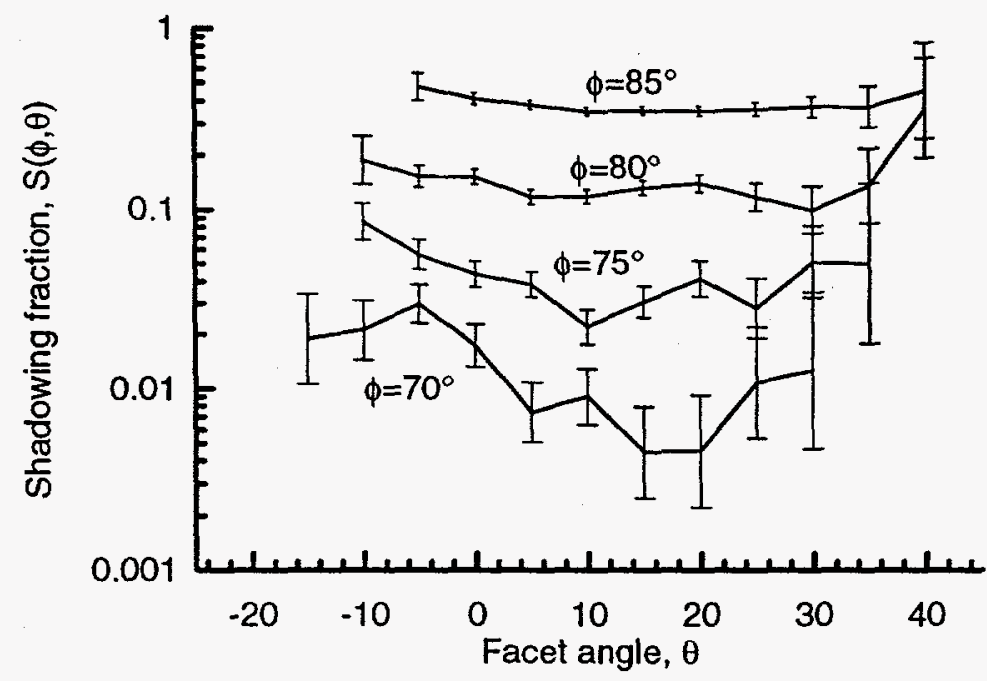

Figure 5. Monte-Carlo estimate of the shadowing fraction is plotted against facet angle. There is some evidence that the fraction does vary with $\theta$.

\section{IMPLICATIONS FOR EMISSIVITY}

It is interesting to ask what implications the purely geometrical constraint in Eq. (17) has on the physical emissivity of a rough surface. In particular, we note that there are two questions which this constraint does not immediately answer:

- Will shadowing tend to increase or decrease the total emissivity of a rough surface?

- Will the effect of multiple reflections dominate or be dominated by the effect of shadowing?

To answer the first question, consider

$$
\Delta \varepsilon(\phi)=\varepsilon_{\text {shadowed }}(\phi)-\varepsilon_{\text {unshadowed }}(\phi)
$$

as the effect of shadowing on emissivity. Here the "shadowed" model is given by Eq. (14) and the "unshadowed" model is the first order model in Eq. (9). (Note that if we take the (invalid) model in Eq. (20) as our unshadowed model, then the effect of shadowing will trivially be to reduce emissivity.) That is,

$$
\begin{aligned}
\Delta \varepsilon(\phi) & =\int_{-\pi}^{\pi} \varepsilon_{o}(\phi-\theta)\left[P_{A}(\phi ; \theta)-P(\phi ; \theta)\right] \mathrm{d} \theta \\
& =-\int_{-\pi}^{\pi} \varepsilon_{o}(\phi-\theta)\left[P_{B}(\phi ; \theta)+P_{C}(\phi ; \theta)\right] \mathrm{d} \theta .
\end{aligned}
$$


We have seen previously that $\int_{-\pi}^{\pi}\left[P_{B}(\phi ; \theta)+P_{C}(\phi ; \theta)\right] \mathrm{d} \theta=0$, so we can write the last equation as:

$$
\Delta \varepsilon(\phi)=\int_{-\pi}^{\pi}\left[1-\varepsilon_{o}(\phi-\theta)\right]\left[P_{B}(\phi ; \theta)+P_{C}(\phi ; \theta)\right] \mathrm{d} \theta
$$

As we can see from Fig. 2, the term $1-\varepsilon_{o}(\phi-\theta)$ is small except as $|\phi-\theta|$ approaches $\pi / 2$, and it is also in this regime that $P_{B}$ generally takes on its largest (absolute) values. By contrast, the $P_{C}$ term is not necessarily largest in this regime, yet on average $P_{C}$ and $P_{B}$ are of equal absolute value. So the integral is dominated by the negative $P_{B}$, and the overall effect of shadowing is to reduce the emissivity of a rough non-Lambertian surface.

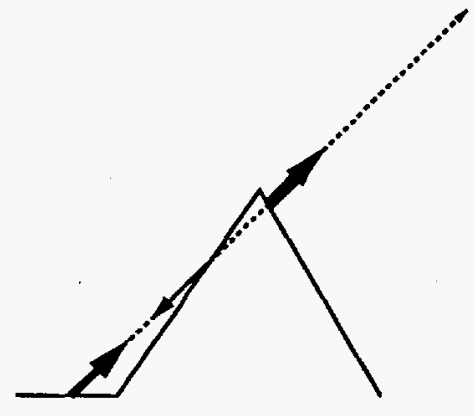

(a)

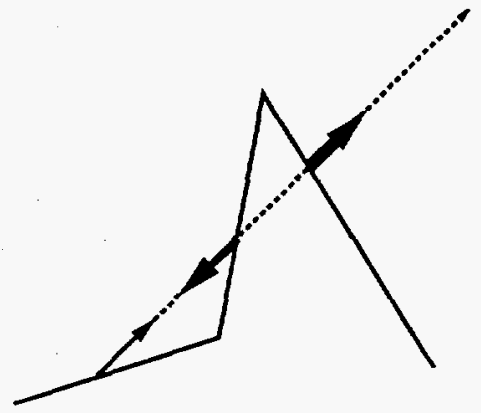

(b)

Figure 6. Two situations in which shadowing alters the total emissivity of the surface with respect to the first-order model. The arrows are taken to be indicative of the strength of the emissivity from each facet. In the first-order model, which neglects shadowing, the total emissivity is the sum of the three emissivities (with the back-angled facet contributing negatively); in the shadowed model, only the emissivity from the ' $\mathrm{A}$ ' facets contributes to the total. In (a), the back-angled facet is steeper than the shadowed facet. Since real (non-Lambertian) emissivity decreases as the angle becomes more grazing, the first-order model (sum of the three arrows) would over-estimate the actual emissivity. In (b), it works the other way around, and the first-order model under-estimates the emissivity. If one believes that situation (a) is more common, then one expects that the effect of shadowing is to reduce the emissivity that would be predicted by the first-order model.

This is a hand-waving argument, however, and one can contrive exceptions. Another way to look at it is illustrated in Fig. 6. Two situations are shown, one (a) for which the effect of shadowing is to reduce the total emissivity with respect to the first-order model, and one (b) for which the effect is to increase emissivity. Which situation has the largest contribution to the total effect depends on the geometry of the surface, and is not determined by $P(\theta)$. However, if intuition (or your favorite surface model) suggests that the first situation (a) is more common, then that implies that the effect is to reduce total emissivity. This is contrary to the folk wisdom that "everything you consider will only raise the emissivity."

Whether this is relevant to real water surfaces, however, depends on the answer to the second question. Shadowing does not occur at all until the look angle becomes steep enough, yet multiple scattering is a phenomenon that occurs even for a vertical look angle. If multiple scattering is more important than shadowing at the more grazing look angles as well, then its effect is to enhance the emissivity of a rough non-Lambertian surface. Experiments with bubbled water surfaces ${ }^{15}$ seem to bear this out. Indeed, the limit of infinite multiple reflections leads to black-body radiation (with effective emissivity given by $\varepsilon=1$ ). More detailed Monte-Carlo ray-tracing simulations ${ }^{12}$ suggest that when the effects of multiple scattering and shadowing are combined, the overall result is to raise the emissivity. Folk wisdom is vindicated.

\section{ACKNOWLEDGEMENTS}

We are grateful to Pierre Villeneuve for useful comments on the manuscript. 


\section{REFERENCES}

1. C. R. Zeisse, "Radiance of the ocean horizon," J. Opt. Soc. Am. A 12, pp. 2022-2030, 1995.

2. J. D. Jackson, Classical Electrodynamics, Second Edition, John Wiley \& Sons, New York, 1975.

3. W. M. Irvine and J. B. Pollack, "Infrared optical properties of water and ice spheres," Icarus 8, pp. 324-360, 1968.

4. However, $P(\phi ; \theta)$ is negative for $|\phi-\theta|>\pi / 2$, so it is not a proper probability.

5. P. M. Saunders, "Shadowing on the ocean and the existence of the horizon," J. Geophys. Res. 72, pp. 4643-4649, 1967.

6. M. D. Mermelstein, E. P. Shettle, E. H. Takken, and R. G. Priest, "Infrared radiance and solar glint at the ocean-sky horizon," Applied Optics 33, pp. 6022-6034, 1994.

7. R. J. Wagner, "Shadowing of randomly rough surfaces," J. Acoust. Soc. Am. 41, pp. 138-147, 1967.

8. It is less general because it assumes that $S(\phi ; \theta)$ is independent of $\theta$.

9. P. M. Saunders, "Radiance of sea and sky in the infrared window $800-1200 \mathrm{~cm}^{-1}$," J. Opt. Soc. Am. 58, pp. $645-652,1968$.

10. C. Cox and W. Munk, "Measurement of the roughness of the sea surface from photographs of the sun's glitter," J. Opt. Soc. Am. 44, pp. 838-850, 1954.

11. I. B. Schwartz and D. Hon, "Emissivity as a function of surface roughness: A computer model," Tech. Rep. NRL Memorandum 5816, Naval Research Laboratory, 1986.

12. B. G. Henderson, J. Theiler, and P. V. Villeneuve, "The polarized emissivity of a wind-roughened sea surface: a Monte-Carlo model," Submitted to Remote Sensing of the Environment, 1997.

13. R. W. Preisendorfer and C. D. Mobley, "Unpolarized irradiance reflectances and glitter patterns of random capillary waves on lakes and seas, by Monte Carlo simulation," NOAA Tech. Memo. ERL PMEL-63, 1985.

14. R. W. Preisendorfer and C. D. Mobley, "Albedos and glitter patterns of a wind-roughened sea surface," J. Phys. Ocean. 16, pp. 1293-1316, 1986.

15. J. R. Schott, B. V. Brower, R. Bhaskar, R. Raqueno, and C. Salvaggio, "Measurement of the optical properties of materials related to ship signatures," Tech. Rep. RIT/DIRS \#90/91-51-140, Digitial Imaging and Remote Sensing Laboratory, Rochester Institute of Technology, 1991. 